\title{
Erratum: Asymptotic Floquet states of non-Markovian systems [Phys. Rev. A 96, 042103 (2017)]
}

Luca Magazzù, Sergey Denisov, and Peter Hänggi

(Q) (Received 24 April 2018; published 3 May 2018)

DOI: 10.1103/PhysRevA.97.059902

The paper contains two typographical errors in Eqs. (6) and (13). The correct Eqs. (6) and (13) read

$$
\mathbf{M}(t)=\left(\begin{array}{cc}
\mathbf{0} & -\mathbf{H}(t) \\
-\mathbf{G}(t) & -\mathbf{A}
\end{array}\right),
$$

and

$$
\mathbf{M}(t)=\left(\begin{array}{cc}
\mathbf{0} & -\mathbf{H} \\
-\mathbf{G}(t) & -\mathbf{A}
\end{array}\right),
$$

respectively (minus signs in the lower-diagonal entries were missing).

As actual calculations have been performed by using the correct expressions for the above matrices, no derivations or results in the remaining parts of the paper are affected by these two typographical errors. 